\title{
Ternary Emission of a Blue-, Green-, and Red-Based Molecular Imprinting Fluorescence Sensor for the Multiplexed and Visual Detection of Bovine Hemoglobin
}

\author{
Qian Yang, ${ }^{\dagger, \ddagger}$ Jinhua Li, ${ }^{\dagger}$ Xiaoyan Wang, ${ }^{\dagger, \S}$ Hua Xiong, ${ }^{*},{ }^{\ddagger}$ and Lingxin Chen ${ }^{*}, \dagger, \|, \perp \odot$ \\ ${ }^{\dagger}$ CAS Key Laboratory of Coastal Environmental Processes and Ecological Remediation, Research Center for Coastal Environmental \\ Engineering and Technology, Yantai Institute of Coastal Zone Research, Chinese Academy of Sciences, Yantai 264003, People's \\ Republic of China \\ ${ }^{\ddagger}$ State Key Laboratory of Food Science and Technology, Nanchang University, Nanchang 330047, People’s Republic of China \\ ${ }^{\S}$ School of Pharmacy, Binzhou Medical University, Yantai 264003, People’s Republic of China \\ "Laboratory for Marine Biology and Biotechnology, Pilot National Laboratory for Marine Science and Technology, Qingdao \\ 13266237, People's Republic of China \\ ${ }^{\perp}$ Center for Ocean Mega-Science, Chinese Academy of Sciences, 7 Nanhai Road, Qingdao 266071, People’s Republic of China
}

\section{Supporting Information}

\begin{abstract}
A novel ternary-emission fluorescence sensor was proposed by post-imprinting mixing blue-/green-/red-emission bovine hemoglobin $(\mathrm{BHb})$ imprinted polymers (b-MIPs, g-MIPs, and r-MIPs) at a proper ratio and realized the multiplexed and visual detection of $\mathrm{BHb}$. The three MIPs were individually embedded with blue-emission 7-hydroxycoumarin, green-emission CdTe quantum dots (QDs), and red-emission $\mathrm{CdTe} / \mathrm{ZnS}$ QDs. Upon interaction with $\mathrm{BHb}$ within $8 \mathrm{~min}$, the fluorescence of CdTe and CdTe/ZnS QDs were simultaneously turned off, whereas the 7-hydroxycoumarin turned on the fluorescence intensity. Thereupon, the ratiometric fluorescence intensity of the ternary emission linearly varied within $0.025-3 \mu \mathrm{M} \mathrm{BHb}$, accompanying the profuse fluorescence color evolution from yellowish green to yellow to salmon to plum to purple to finally blue. In comparison with the dual- or single-emission sensor, the ternary-emission fluorescence MIPs sensor provided a wider color variation covering the green-red-blue window for accurate naked-eye determination of $\mathrm{BHb}$, as well as a lower detection limit down to $7.8 \mathrm{nM}$ and a higher imprinting factor of 15.2. Moreover, the satisfactory recoveries of $99.25-111.7 \%$ in determining the spiked $\mathrm{BHb}$ in bovine urine samples, as well as the optical stability and post-imprinting construction convenience, indicated that the developed tricolor-emission fluorescence MIPs sensor provided an ideal alternative for rapid, sensitive, and visual determination of proteins in complicated samples.

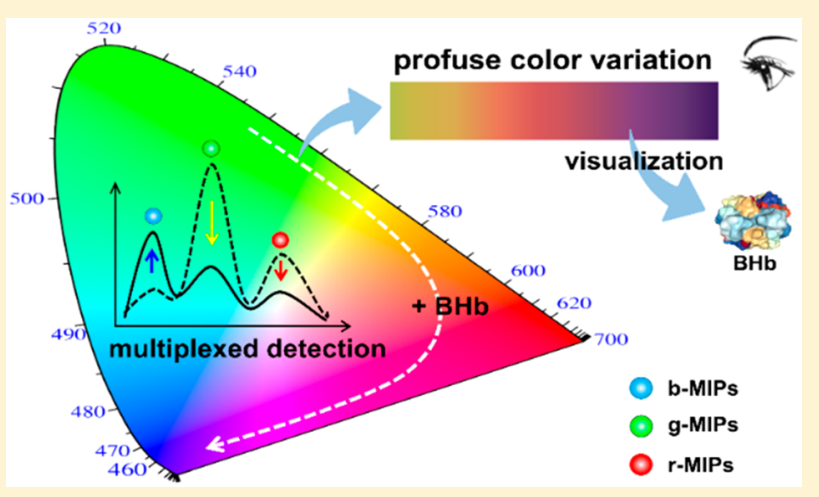

C urrently, rapid visual detection plays a significant role in environmental monitoring, food security, clinical diagnostics, therapeutics, and so forth. For the mass samples, a complete detection program is always constituted by rapid rudimentary on-site monitoring and frontline screening and the further confirmation of suspected species by a large-sized precision instrument such as liquid chromatography-mass spectrometry. The rapid monitoring and screening greatly reduces the workload and test expense; specifically, its fundamental purpose is to selectively recognize target analytes with effective discrimination against coexisting substances and to reduce a false positive. As is known, various natural ligands can provide recognition specificity to species such as antibodies, ${ }^{1}$ antimicrobial peptides, ${ }^{2}$ sugars/lectins, ${ }^{3}$ phages, ${ }^{4}$ aptamers, ${ }^{5}$ etc. However, the lack of operation flexibility, physical/chemical stability, and economic efficiency has promoted researchers to explore more applicable and economical methods, such as molecular imprinting technology.

In brief, molecular imprinting is a method to prepare molecularly imprinted polymers (MIPs), through polymerizing the template molecules into the imprinting matrix and then removing templates. The as-left imprinted cavities can always selectively recognize and rebind the target analytes since they are complementary in shape, size, and functional groups. ${ }^{6,7}$ In comparison with the aforementioned natural counterparts, MIPs are inexpensive and can withstand harsher environmental conditions. ${ }^{8}$ Up to now, MIPs toward small molecules have

Received: January 7, 2019

Accepted: April 23, 2019

Published: April 23, 2019 
been easily proposed, whereas the imprinting for biomacromolecules such as proteins is still a significant challenge. Flexible conformation and easy denaturation require harsh imprinting conditions close to those of the biomacromolecule natural environments, and the large size results in restricted mobility within highly cross-linked MIPs and poor eluting/ rebinding efficiency." ${ }^{9,0}$ Surface imprinting in buffer solution, an alternative solution, can locate the imprinted cavities at or in the proximity of the surface, reducing the mass transfer resistance and increasing the cavity accessibility. ${ }^{9,10}$

By virtue of their endogenous superselectivity, MIPs combined with proper signal transducers have been widely applied in rapid testing. ${ }^{7}$ Among them, molecular imprinted fluorescence sensors have been brought to the front of the search for optical sensors thanks to their high sensitivity of fluorescence detection. By a general survey, ${ }^{11}$ we have observed that, in the early stage, these sensors always focus on the analyte-dependent fluorescence intensity change in a "single-emission" mode. That is to say, they can only emit one fluorescence peak and the brightness can be enhanced ${ }^{12}$ or quenched $^{13}$ upon analyte recognition. However, a variety of analyte-independent factors such as background fluorescence, instrumental fluctuation, and operational parameters can cause the fluctuation of the fluorescence spectra, and a slight fluorescence brightness variation is difficult to catch by the naked eye. ${ }^{11}$ Lately, two individual fluorescence substances have been introduced into MIPs preparation, endowing the asobtained sensors with dual fluorescence peaks (bimodal fluorescence) at a single excitation wavelength. The two fluorescence intensities would not change synchronously upon the analyte recognition, so to possess a self-correction function and fluorescence color variation. ${ }^{14}$ For example, when a greenand red-emission MIPs sensor reacted with targeted analyte, the red fluorescence was quenched while the green remained constant, changing the fluorescence color from red to yellow to green, ${ }^{15}$ as schematically illustrated in Figure 1 . The

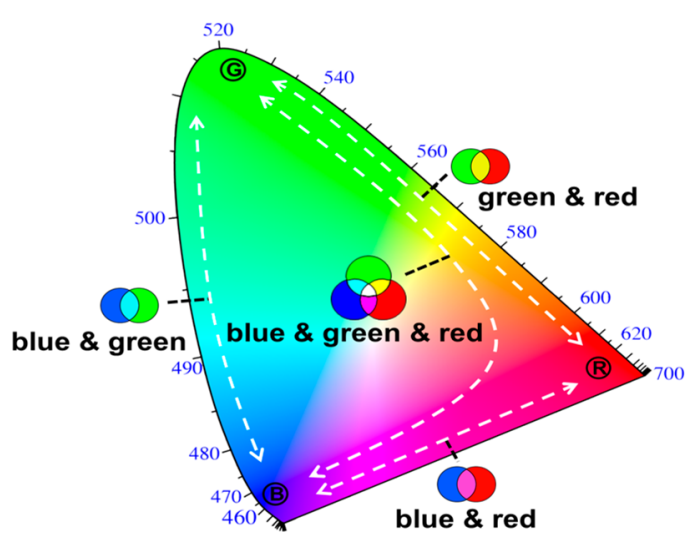

Figure 1. Chromaticity diagram to indicate the range of color variation.

cooperation of two fluorescence colors, such as blue mixed with red $^{16}$ or blue mixed with green ${ }^{17}$ also presented color evolution of the blue-red or blue-green window, as indicted in the chromaticity diagram (Figure 1). In comparison with the brightness change, color variation is more easily caught; however, the color-variation range is still compressed. A tricolor-emission system based on a red-green-blue (RGB)fluorescence mode can further expend the color evolution range and exhibit more profuse fluorescence color within the green-yellow-red-purple-blue window (including their transitional colors), ${ }^{18}$ as displayed in Figure 1, but this process remains immature in analytical sciences and is untouched in molecular imprinting fluorescence sensor construction. Obviously, for multiemission sensor construction, semiconductor quantum dots (QDs) are a fantastic alternative due to their unique optical properties: (i) wide excitation spectra ensure that QDs are simultaneously excited by the excited light for other fluorescence materials, (ii) narrow symmetrical emission profiles benefit their separation from other peaks especially in multiplexed detection, and (iii) a large Stokes shift and controllable emission wavelength favor various fluorescence color selections and cooperations. ${ }^{19}$

Hence, as a proof of concept, we intend to propose a tricolor-emission (blue-, green-, and red-emission) fluorescence MIPs sensor for selective protein detection, taking bovine hemoglobin $(\mathrm{BHb})$ as a model protein. Blue-emission 7-hydroxycoumarin, green-emission CdTe (g-CdTe) QDs, and red-emission $\mathrm{CdTe} / \mathrm{ZnS}$ (r-CdTe/ZnS) QDs were separately embedded into $\mathrm{BHb}$ imprinted polymers via a sol-gel method and used as response signals to provide blue, green, and red fluorescence, respectively. A tricolor-emission sensing system was constructed by simply mixing the as-obtained blue-, greenand red-emission MIPs (namely b-MIPs, g-MIPs, and r-MIPs for simplicity) in an optimized proportion. The multimodal sensing platform has both the high selectivity of MIPs and the high sensitivity as well as the self-correction function of multiplexed fluorescence detection. Upon $\mathrm{BHb}$ recognition, blue fluorescence was enhanced while the green and red fluorescence was quenched, leading to the ratiometric fluorescence intensities of $I_{\text {blue }} / I_{\text {green }} / I_{\text {red }}$ gradually increasing with increasing $\mathrm{BHb}$. Significantly, the intensity ratio variation could be directly expressed as the fluorescence color evolution from yellowish green to yellow to salmon to plum to purple to finally blue, allowing the naked-eye visual detection of $\mathrm{BHb}$. It was demonstrated that the sensitivity, visualization, and operational efficiency of the tricolor-emission fluorescence MIPs sensor were significantly enhanced, through integration of surface imprinting, tricolor-emission fluorescence, and postimprinting mixed-type construction together.

\section{EXPERIMENTAL SECTION}

Materials and Chemicals. $\mathrm{BHb}$, bovine serum albumin (BSA), and Triton X-100 were purchased from Solarbio Science \& Technology Co. Ltd. (Beijing, China). Lysozyme was offered by Sigma-Aldrich (Shanghai, China). (3Aminopropyl)triethoxysilane (APTES), tetraethyl orthosilicate (TEOS), 7-hydroxycoumarin, and ovalbumin were supplied by Aladdin (Shanghai, China). Ammonium hydroxide $\left(\mathrm{NH}_{3}\right.$. $\mathrm{H}_{2} \mathrm{O}$ ) and ethanol were provided by Sinopharm Chemical Reagent Co. Ltd. (Shanghai, China). All reagents were at least of analytical grade and were used as received. PBS solution (0.01 M, pH 7.0) were used for MIPs preparation and sensing procedures, and ultrapure water of $18.2 \mathrm{M} \Omega$ specific resistance (Millipore, USA) was used in all experiments.

Instrumentation. A Fluoromax-4 spectrofluorometer (Horiba Scientific) and Thermo Scientific NanoDrop 2000/ 2000C spectrophotometer (Thermo Fisher Scientific) were used to record fluorescence emission and UV-vis absorption spectra, respectively. Morphological evaluation was examined on a scanning electron microscope (SEM, JSM-6701F) and a transmission electron microscope (TEM, JEM-2100). Elemental spectra were observed by energy dispersive spectros- 
copy (EDS, JSM-6701F). FT-IR analyses were performed on a FT-IR spectrometer (Thermo Nicolet Corp.).

Preparation of Core-Shell Structured B-, G-, and REmission $\mathrm{BHb}$ Imprinted Nanoparticles. $\mathrm{SiO}_{2}$, g-CdTe, and $\mathrm{r}-\mathrm{CdTe} / \mathrm{ZnS}$ QDs were first synthesized according to previous studies ${ }^{20-22}$ with necessary modification, respectively (with synthesis details given in the Supporting Information). Then, core-shell structured blue-emission MIPs (b-MIPs) were prepared by embedding 7-hydroxycoumarin in the imprinting shell to provide blue fluorescence via a typical sol-gel procedure. Briefly, $10 \mathrm{mg}$ of $\mathrm{BHb}$ and $35 \mu \mathrm{L}$ of APTES were added to $20 \mathrm{~mL}$ of PBS solution containing $20 \mathrm{mg}$ of 7hydroxycoumarin and $10 \mathrm{mg}$ of $\mathrm{SiO}_{2}$ nanoparticles. After the mixture was stirred for $1 \mathrm{~h}$ to form the BHb-APTES complex, $56 \mu \mathrm{L}$ of $\mathrm{NH}_{3} \cdot \mathrm{H}_{2} \mathrm{O}$ and $56 \mu \mathrm{L}$ of TEOS were added and the mixture was stirred for $12 \mathrm{~h}$ in the dark. Finally, the obtained imprinting matrix was eluted six times using $0.5 \%$ Triton X-100 solution to remove $\mathrm{BHb}$ and dispersed into $20 \mathrm{~mL}$ of $\mathrm{PBS}$ solution for further experiments. As a control, blue-emission nonimprinted polymers (b-NIPs) were synthesized using the same procedure but without adding $\mathrm{BHb}$. Likewise, green- and red-emission MIPs (g-MIPs, r-MIPs) and their corresponding NIPs (g-NIPs, r-NIPs) were prepared according to the b-MIPs and b-NIPs preparation procedure but with 7-hydroxycoumarin replaced by $3 \mathrm{~mL}$ of $\mathrm{g}-\mathrm{CdTe}$ and $12 \mathrm{~mL}$ of $\mathrm{r}-\mathrm{CdTe} / \mathrm{ZnS}$ QDs, respectively, with the final volume brought to $20 \mathrm{~mL}$ by readjusting PBS solution.

Construction of the $\mathrm{BHb}$ Imprinted Tricolor-Emission Sensor. For optimal $\mathrm{BHb}$ visualization, the tricolor sensor was constructed by thoroughly dispersing b-MIPs (5\%, v/v), gMIPs $(11 \%, \mathrm{v} / \mathrm{v})$, and $\mathrm{r}-\mathrm{MIPs}(6 \%, \mathrm{v} / \mathrm{v})$ into BHb-containing PBS solution, where the final concentration of $\mathrm{BHb}$ ranged from 0 to $3 \mu \mathrm{M}$. After incubation for $8 \mathrm{~min}$, the fluorescence spectra were recorded under a single excitation wavelength of $365 \mathrm{~nm}$ with slit widths of $5 / 5 \mathrm{~nm}$. The corresponding fluorescence images were observed under a $365 \mathrm{~nm}$ UV lamp. The selectivity of the constructed tricolor-emission sensor toward $\mathrm{BHb}$ was evaluated by comparing its fluorescence change efficiency and fluorescence image toward other proteins at the same concentration of $1 \mu \mathrm{M}$.

Analysis of Real Samples. Two bovine urine samples from healthy bovine were kindly provided by a local butcher, and the possible $\mathrm{BHb}$ in the samples was first analyzed by a hemoglobin detection meter (BHb enzyme-linked immunosorbent assay test kit or urine tester). After they were filtered with a $0.22 \mu \mathrm{m}$ microfiltration membrane, the 100-fold diluted urine samples with PBS solution were used to perform the recovery experiments at four concentration levels of 0.050 , $0.250,1.000$, and $2.000 \mu \mathrm{M} \mathrm{BHb}$ using the constructed tricolor sensor, respectively. All tests were performed in triplicate.

\section{RESULTS AND DISCUSSION}

Construction and Characterization of the $\mathrm{BHb}$ Imprinted Tricolor-Emission Sensor. In the present work, 7-hydroxycoumarin, g-CdTe, and r-CdTe/ZnS QDs were used to prepare fluorescent MIPs and to provide blue, green, and red fluorescence, respectively. As illustrated in Figure 2A, taking the b-MIPs preparation as an example, surface imprinting was conducted on the surface of $\mathrm{SiO}_{2}$ nanoparticles, using 7-hydroxycoumarin, $\mathrm{BHb}$, APTES, TEOS, and $\mathrm{NH}_{3}$. $\mathrm{H}_{2} \mathrm{O}$ as fluorescence provider, imprinted template, functional monomer, cross-linker, and catalyst, respectively. After the sol-gel polymerization, $\mathrm{BHb}$ molecules were eluted to obtain

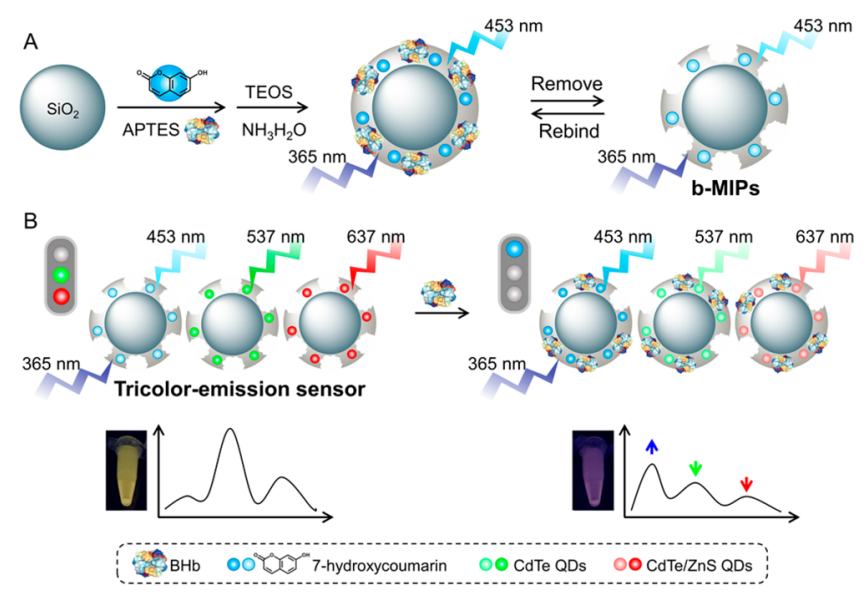

Figure 2. Schematic illustration for (A) the preparation of blueemission MIPs (b-MIPs) and (B) the construction of a tricoloremission sensor by mixing blue-, green- and red-emission MIPs (b-, $\mathrm{g}-$, and r-MIPs) at an optimized volume ratio of 50/110/60.

the b-MIPs with selective imprinted cavities complementary to $\mathrm{BHb}$ in shape, size, and functional groups. Likewise, g-MIPs and r-MIPs were obtained following the same procedure but replacing 7-hydroxycoumarin with g-CdTe and $\mathrm{r}-\mathrm{CdTe} / \mathrm{ZnS}$ QDs, respectively. After the three individual single-emission MIPs were mixed in the proper ratio of 50/110/60 (v/v/v), the as-constructed tricolor sensor exhibited multimodal emission at 453, 537, and $637 \mathrm{~nm}$ along with yellowish green fluorescence under a single $365 \mathrm{~nm}$ excitation (Figure $3 \mathrm{~A})$. Upon the $\mathrm{BHb}$ recognition by the recognition sites in the ultrathin MIPs, a simultaneous multiplexed variation of "turnon" blue fluorescence but "turn-off" green and red fluorescence appeared, changing the fluorescence color to purple or even blue (Figure 2B).

The morphologies of CdTe, CdTe/ZnS QDs, $\mathrm{SiO}_{2}$, and b-, g-, and r-MIPs and their corresponding NIPs were investigated by SEM and TEM. As observed in Figure S1, CdTe and $\mathrm{CdTe} / \mathrm{ZnS}$ QDs had average diameters of approximately 2 and $5 \mathrm{~nm}$, respectively. As displayed in Figure 3B and Figure S2A, $\mathrm{SiO}_{2}$ nanoparticles possessed a smooth surface and monodisperse size with an average diameter of about $79 \mathrm{~nm}$, whereas b-, g-, and r-MIPs and NIPs shared a rough polymeric surface and larger particle size of approximately $95 \mathrm{~nm}$ (Figure 3C-H and Figure $S 2 B-G$ ). The small particle sizes endowed the MIPs composites with good dispersibility and stability in an aqueous solution, avoiding aggregation and precipitation during the fluorescence measurement. In addition, as estimated, the imprinting layer thickness was controlled to be approximately $8 \mathrm{~nm}$, similar to the diameter of the protein template molecules, so to guarantee that all the imprinted cavities were located at the surface or in the proximity of the MIPs surface, providing higher cavity accessibility and lower mass transfer resistance. ${ }^{23}$ Common elements of $\mathrm{C}, \mathrm{O}$, and $\mathrm{Si}$ were observed in b-, g-, and r-MIPs (Figure S3), indicating the basic $\mathrm{SiO}_{2}$ matrices of MIPs prepared by sol-gel polymerization. Additionally, g-MIPs contained S, Cd, and Te (Figure $\mathrm{S3B}$ ), belonging to the sulfhydryl groups capped CdTe QDs, and $\mathrm{r}$-MIPs possessed $\mathrm{Cd}, \mathrm{Te}, \mathrm{Zn}$, and $\mathrm{S}$ elements (Figure $\mathrm{S} 3 \mathrm{C}$ ), demonstrating the successful embedding of $\mathrm{CdTe} / \mathrm{ZnS}$ QDs. Specifically, the $\mathrm{N}$ element of functional groups and GSH disappeared because of the absorbance by the adjacent $\mathrm{C}$. 

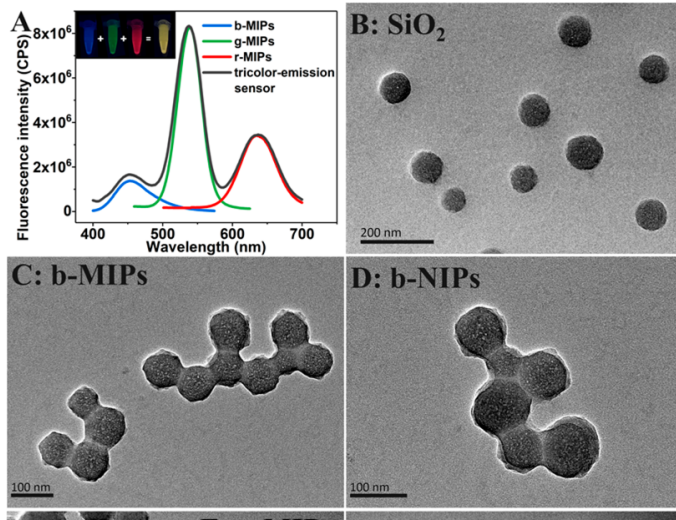

D: b-NIPs
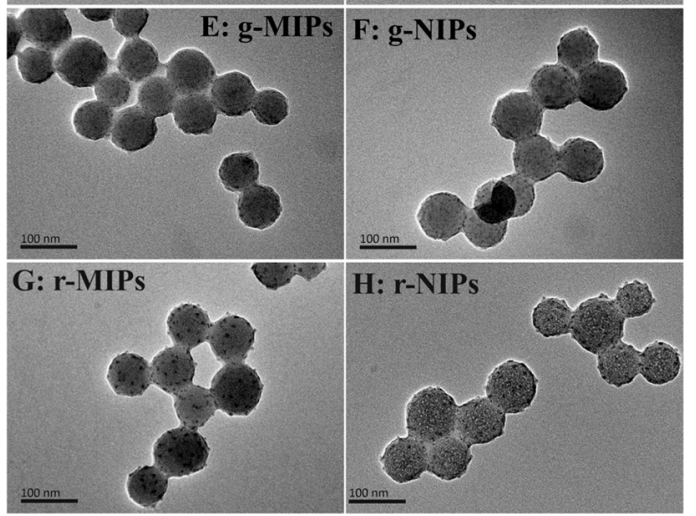

Figure 3. (A) Fluorescence emission spectra and images of b-, g-, and r-MIPs and the tricolor-emission MIPs sensor under a single $365 \mathrm{~nm}$ excitation, respectively, and TEM images of (B) $\mathrm{SiO}_{2}$ nanoparticles and $(\mathrm{C}, \mathrm{E}, \mathrm{G}) \mathrm{b}-$-, g-, and r-MIPs and $(\mathrm{D}, \mathrm{F}, \mathrm{H})$ the corresponding b-, $\mathrm{g}-$,and $\mathrm{r}$-NIPs.

Furthermore, the functional groups of the $\mathrm{SiO}_{2}$ and MIPs/ NIPs were observed by FT-IR spectra (Figure S4). As can be seen, MIPs and NIPs shared the similar primary compositions with $\mathrm{SiO}_{2}$ nanoparticles, since there is no obvious difference in their major backbones: characteristic peaks at 461 and 790 $\mathrm{cm}^{-1}$ belonged to the stretching vibration of $\mathrm{Si}-\mathrm{O}$, and the strong band at $1095 \mathrm{~cm}^{-1}$ corresponded to the stretching vibration of $\mathrm{Si}-\mathrm{O}-\mathrm{Si}$. The wide absorption band at $3455 \mathrm{~cm}^{-1}$ could be ascribed to the $\mathrm{O}-\mathrm{H}$ stretching vibration of $\mathrm{SiO}_{2}$ and MIPs/NIPs, as well as the stretching vibration of $\mathrm{N}-\mathrm{H}$ of APTES. In comparison with $\mathrm{SiO}_{2}$, the appearance of a 1388 $\mathrm{cm}^{-1}$ peak in MIPs and NIPs could be attributed to the vibrations of $\mathrm{C}-\mathrm{O}$, which may be from TGA, GSH, and 7hydroxycoumarin, demonstrating the successful introduction of fluorescent substances.

All the above characterization results confirmed that the fluorescence substances were successfully introduced into the MIPs/NIPs layer coated on the $\mathrm{SiO}_{2}$ surface.

Control of Key Points for the Tricolor MIPs Sensor Construction. Each experiment has its own specific parameters to determine the final performance. Three key parameters were necessary to be optimized in this work, namely (i) separating 7-hydroxycoumarin from QDs in the MIPs preparation, (ii) expanding the difference in quenching rates between green- and red-emission peaks, and (iii) finding the appropriate ratio in fluorescence intensity of the three emission peaks.

As seen in Figure S5A, when the ternary-emission MIPs sensor was prepared by a one-pot imprinting method of mixing 7-hydroxycoumarin, g-CdTe, and r-CdTe QDs simultaneously, the fluorescence quenching degree of the green and red fluorescence response to $1 \mu \mathrm{M} \mathrm{BHb}$ was unsatisfactory, less than $29.1 \%$ and $25.4 \%$, respectively; what was worse, the blueemission peak was out of shape and exhibited a intensity decrease toward $\mathrm{BHb}$. The simultaneous quenching of the three peaks would cause a narrow color variation and weak fluorescence intensity with $\mathrm{BHb}$ recognition. Likewise, for the two dual-emission MIPs prepared by a one-pot method, i.e., emitting blue-green and blue-red fluorescence, respectively, there was not only slight quenching of 7-hydroxycoumarin and QD emission but also the appearance of a malformed blue-
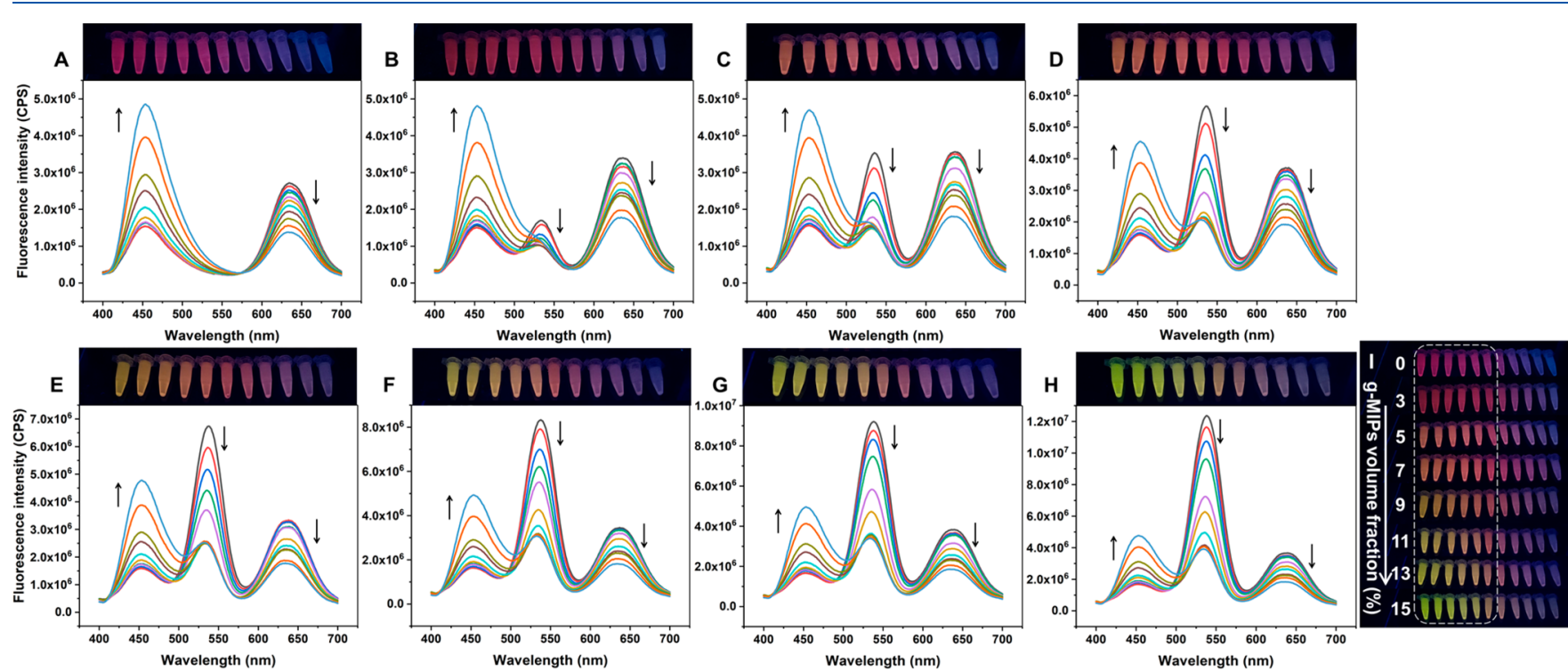

Figure 4. Fluorescence spectra of tricolor-emission MIPs sensor for BHb detection from 0 to $3 \mu \mathrm{M}$ as indicated by the direction of the arrows, where the sensor contained 5\% b-MIPs, 6\% r-MIPs, and (A) 0, (B) 3\%, (C) 5\%, (D) 7\%, (E) 9\%, (F) 11\%, (G) 13\%, and (H) 15\% g-MIPs, respectively. The upper insets give the corresponding fluorescence images under a $365 \mathrm{~nm}$ UV lamp. (I) Combination of the fluorescence color images for a more intuitional color comparison. 
emission peak in the sensing performance study (Figure S5B,C). It could be assumed that 7-hydroxycoumarin and QDs may interfere with each other. In addition, in comparison with the traditional one-pot method, ${ }^{24}$ the post-imprinting mixing method $^{25}$ can simplify the optimization of emission intensity ratio and thereby shorten the experimental cycle and save cost. Hence, the b-MIPs, g-MIPs, and r-MIPs were first prepared, respectively, and then were mixed at an appropriate ratio for further multiplexed $\mathrm{BHb}$ detection.

On the basis of the post-imprinting mixing method, an unceasing failure still occurred in obtaining a profuse color variation when the $\mathrm{r}-\mathrm{CdTe} \mathrm{QDs}$ were used to provide the red fluorescence. As shown in Figure S6A, the fluorescence quenching rates of green and red peaks were calculated, where the g-CdTe@MIPs and r-CdTe@MIPs were mixed to detect $\mathrm{BHb}$. A similar quenching step was observed that the two fluorescence peaks were both rapidly quenched by ultralow-concentration $\mathrm{BHb}$ and then slowly quenched by higher-concentration $\mathrm{BHb}$, because of the gradual occupation and finally almost saturation of the imprinted cavities. Concomitantly, the fluorescence color changed from bright yellow to pale yellow to finally dim red (inset of Figure S6B). When the blue MIPs participated in the sensing procedure, the cooperation of enhancing blue fluorescence only produced a yellow-blue color evolution (inset of Figure S6C). Hence, we tried to use $\mathrm{r}-\mathrm{CdTe} / \mathrm{ZnS}$ QDs to provide red fluorescence. Due to the $\mathrm{ZnS}$ shell protection, the sensitivity of r-MIPs toward $\mathrm{BHb}$ was greatly reduced (Figure S6D). The obvious difference in quenching rates made the fluorescence color of the dual-emission sensing system vary from yellowish green to yellow to orange to red with a total intensity decrease (Figure S6E). Excitingly, by a combination with brighter blue fluorescence, the tricolor-emission sensor could provide a profuse fluorescence color in the yellow-salmon-plumpurple-blue range (inset of Figure S6F). Hence, r-CdTe/ ZnS@MIPs were used in further experiments.

The original fluorescence intensity ratio of the three peaks also decided the visualization effect, and the original fluorescence intensity ratio depended on the volume fraction of b-, g-, and r-MIPs. The b-MIPs and r-MIPs were fixed at 5\% and 6\%, respectively; an increase in blue fluorescence and decrease in red fluorescence caused by $\mathrm{BHb}$ recognition were exhibited in the fluorescence spectra with color variation from purplish red to blue (Figure 4A). With an increase in the fraction of g-MIPs from 3 to $15 \%$ (Figure $4 \mathrm{~B}-\mathrm{H}$ ), the original color of the tricolor-emission sensor changed from purplish red to orange to yellow to olive drab. An excessively low g-MIPs dosage could not endow the fluorescence color evolution with a yellow window (Figure 4A-E), while superabundant g-MIPs would cover the red fluorescence, making the color evolution short of the red part (Figure 4G,H). A more intuitional comparison of color variation is given in Figure 4I, where the dotted line circles the color change by trace $\mathrm{BHb}$ from 0 to 250 $\mathrm{nM}$, and a relatively wide variation from yellowish green to yellow to salmon to plum was obtained; after recognition of $\mathrm{BHb}$ at relatively high concentrations, the color would further change to purple and blue when the dosage of g-MIPs was fixed at $11 \%$ (Figure $4 \mathrm{~F}$ ). Hence, $11 \% \mathrm{~g}$-MIPs were used in the ternary-emission MIPs sensor construction.

Multiplexed Detection of $\mathrm{BHb}$ by the Multimodal MIPs Sensor. The response time of the tricolor-emission MIPs sensor toward $\mathrm{BHb}$ was first investigated prior to the sensitivity study. As shown in Figure S7, the fluorescence intensity ratiometric values of $I_{\text {blue }} / I_{\text {green }} / I_{\text {red }}$ (with calculation details given in the Supporting Information) immediately increased with the addition of $1 \mu \mathrm{M} \mathrm{BHb}$ and reached a plateau within $8 \mathrm{~min}$. That is, the composited sensor took a certain time of less than $8 \mathrm{~min}$ to reach a dynamic equilibrium upon the selective recognition of $\mathrm{BHb}$. Such a rapid response was attributed to the ultrathin MIPs layer and the improved site accessibility obtained by the surface imprinting strategy. Therefore, $8 \mathrm{~min}$ was set for the MIPs sensor exposure to the $\mathrm{BHb}$ analyte solution in order to attain accurate, reliable, and stable fluorescence response properties. Possibly, reducing the MIP-shell thickness, providing the (meso)porous structures for MIPs, and improving the diffusion/mixing efficiency of $\mathrm{BHb}$ into MIPs are three effective ways to make this time shorter, since they could greatly facilitate mass transfer and cavity accessibility.

The sensitivity performance of the as-constructed tricoloremission MIPs sensor was investigated by observing its ratiometric fluorescence intensities and color change toward 0-3 $\mu \mathrm{M} \mathrm{BHb}$ (Figure 5A). Before $\mathrm{BHb}$ recognition, the tricolor sensor emitted three fluorescence peaks centered at 453, 537, and $637 \mathrm{~nm}$ under a single excitation; after recognition through hydrogen bonds, the green and red

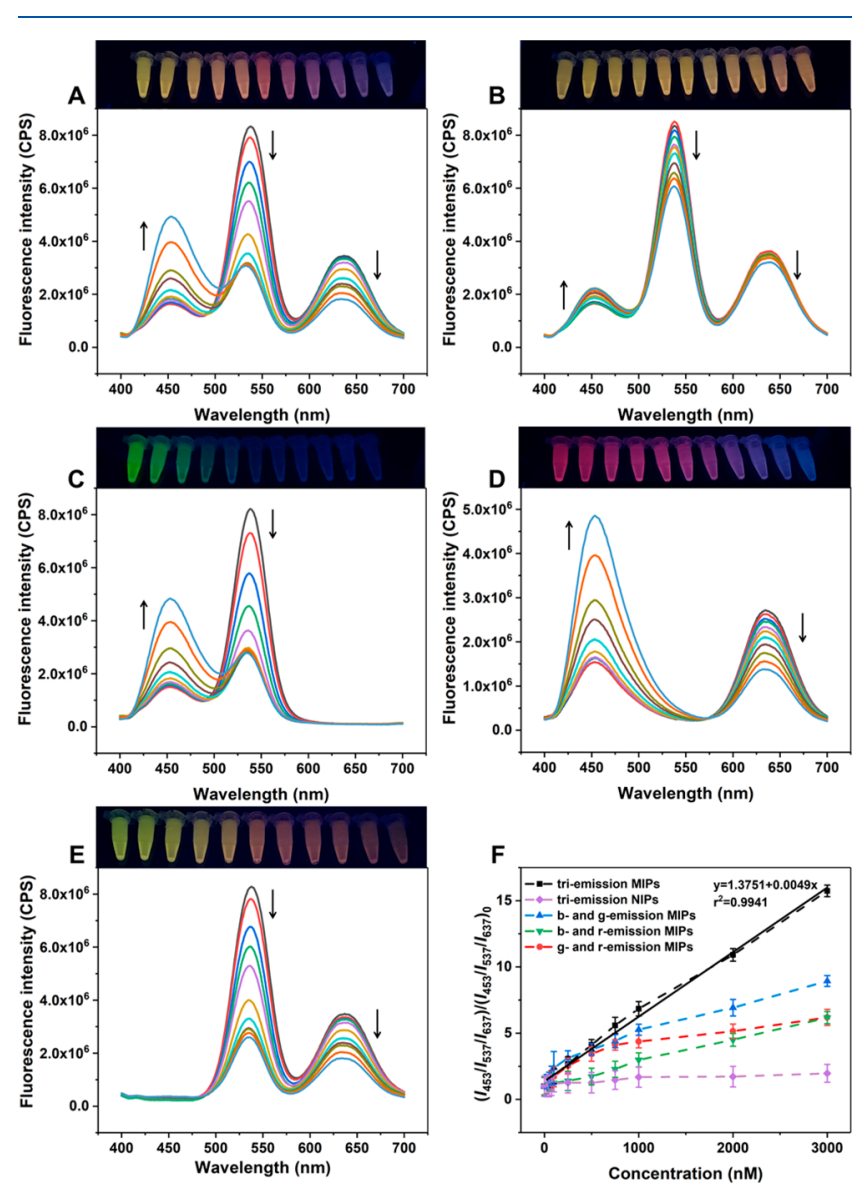

Figure 5. Fluorescence spectra of (A) ternary-emission MIPs sensor, (B) ternary-emission NIPs sensor, and (C) b-, g-MIPs, (D) b-, rMIPs, and (E) g-, r-MIPs sensor upon the recognition of $\mathrm{BHb}$ at increasing concentrations of $0,0.025,0.05,0.075,0.1,0.25,0.5,0.75$, 1,2 , and $3 \mu \mathrm{M}$ as indicated by the direction of the arrows, respectively. (F) Corresponding fluorescence intensity ratio changes. The upper insets are the corresponding fluorescence images with increasing $\mathrm{BHb}$ addition from left to right. 
fluorescence peaks were gradually quenched while the blueemission peak was enhanced. Thanks to the simultaneous fluorescence intensity increasing and decreasing, even a slight intensity change (caused by trace $\mathrm{BHb}$ ) of the three peaks resulted in a distinguishable and profuse color evolution, ranging from the original yellowish green to yellow to salmon to plum to purple and finally to blue under a $365 \mathrm{~nm}$ UV lamp (upper inset of Figure 5A), which was easily caught by the naked eye. The change in fluorescence intensity ratio of $\left(I_{453} /\right.$ $\left.I_{537} / I_{637}\right) /\left(I_{453} / I_{537} / I_{637}\right)_{0}$ with the $\mathrm{BHb}$ concentration is illustrated in Figure 5F, showing a linear increase within $0.025-3 \mu \mathrm{M} \mathrm{BHb}$ and a correlation coefficient $\left(r^{2}\right)$ of 0.9941 . An ultralow limit of detection (LOD) of $7.8 \mathrm{nM}$ was obtained on the basis of the $3 \sigma$ IUPAC criteria of $3 \sigma / s$, where $\sigma$ is the standard deviation of the blank measurements and $s$ is the slope of the linear calibration. A fluorescence resonance energy transfer (FRET) mechanism could be excluded for the quenching route because there was no overlap between the absorbance of $\mathrm{BHb}$ and the fluorescence emission of the $\mathrm{g}$ $\mathrm{CdTe}$ and $\mathrm{r}-\mathrm{CdTe} / \mathrm{ZnS}$ QDs (Figure S8). It was inferred that the photoinduced electron transfer (PET) process between the excited $\mathrm{QDs}$ and the $\mathrm{BHb}$ in a nonradioactive decay pathway caused the fluorescence quenching. Under photoexcitation, hemeproteins(III) can directly intercept one of the charge carriers and be reduced to hemeproteins(II), therefore causing the fluorescence quenching of QDs. ${ }^{26,27}$ In addition, it was assumed that the $\mathrm{BHb}$ binding changed the hydrophobic/ hydrophilic conditions of the imprinted cavities and, therefore, the fluorescence intensity of 7-hydroxycoumarin was enhanced. $^{28}$

In contrast, the fluorescence intensity ratio and color variation toward $0-3 \mu \mathrm{M} \mathrm{BHb}$ of the tricolor-emission NIPs sensor were overshadowed (Figure 5B). Due to the similar shape and size of MIPs and NIPs, it was believed that the difference in recognition behavior could not be attributed to the morphological difference but to the imprinting effect. No selective recognition sites existed in the NIPs, and the small intensity changes were attributed to nonspecific recognition. Such different results between MIPs and NIPs could well demonstrate the high efficiency of the imprinting process. ${ }^{26}$ Furthermore, the imprinting factor, defined as the linear slope ratio of MIPs to NIPs, was calculated to be as high as 15.2, indicating the high imprinting effect of the ternary-emission MIPs (Figure 5F).

The superiority of the ternary-emission MIPs sensor in sensitivity and visualization was confirmed by comparing it with the binary- and single-emission sensors. As can be seen, for the dual-emission sensors of b-,g-MIPs (Figure 5C) as well as b-,r-MIPs (Figure 5D) toward $\mathrm{BHb}$, similar blue fluorescence increases and green/red fluorescence quenching were observed, resulting in a color change from the original green or purple to blue. However, for the g-,r-MIPs (Figure $5 \mathrm{E}$ ), only fluorescence quenching was observed in both peaks, resulting in a fluorescence color change from yellowish green to orange red. In addition, the ratiometric fluorescence intensity changes for the three dual-emission sensors (the slopes in Figure 5F) were significantly lessened, indicating the lower sensitivity. For the single-emission sensors of b-MIPs, gMIPs, and r-MIPs, only brightness change of "turn-on" or "turn-off" mode appeared (Figure S9A-C), with negligibly higher fluorescence enhancement of b-MIPs and quenching of g-MIPs and r-MIPs in comparison to those in mixed use because the three MIPs would share $\mathrm{BHb}$ templates of a certain concentration (Figure S9D-F). Hence, we can draw the conclusion that the multiplexed fluorescence MIPs sensor won out in both the sensitivity and visualization.

The selectivity of the tricolor-emission MIPs sensor was investigated by cross-reactivity experiments toward $\mathrm{BHb}$ and the proteins of BSA, ovalbumin, and lysozyme at the same concentrations. As seen in Figure 6, for MIPs, BHb caused a

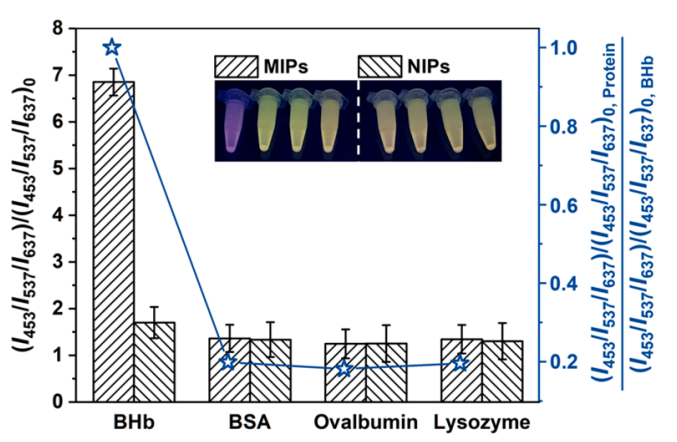

Figure 6. Fluorescence intensity ratios (left) and images (inset) of the tricolor-emission MIPs/NIPs sensors after recognition of BHb, BSA, ovalbumin, and lysozyme at the same concentration of $1 \mu \mathrm{M}$ and fluorescence intensity ratios (right, and blue stars) from the calibration plot for a given protein to that for $\mathrm{BHb}$.

significant increase in fluorescence intensity ratio to evolve the original yellowish green into purple fluorescence; conversely, the other proteins only generated slight fluorescence intensity changes (approximately $19 \%$ of that for $\mathrm{BHb}$ as displayed by blue stars), so that the fluorescence color change was hard to catch by the naked eye. The high selectivity was derived from the designed geometric and dynamic features of the MIPs, which were exclusively complementary for the desired $\mathrm{BHb}$ rather than other possible interfering substances. Meanwhile, the NIPs sensor had little interaction with these proteins and no fluorescence color change could be distinguished, because no selective recognition sites toward any proteins were located within the polymerization layer of NIPs.

Another sensing performance of stability was also investigated by repeatedly measuring the fluorescence spectra and then observing the fluorescence intensity ratio change every 10 min after $\mathrm{BHb}$ addition. As seen in Figure S10A, the fluorescence intensity ratio change remained stable within 60 min. In addition, the fluorescence intensity during storage was also measured every day and the sensor retained $95 \%$ of its original response (Figure S10B). Both of these facts indicated that the tricolor-emission MIPs sensor had excellent selectiverecognition stability and acceptable storage stability, which might be due to the protection of the highly cross-linked imprinting layer.

Practical Applicability and Method Performance Comparison. Recovery experiments in two real samples of bovine urine were conducted with the developed tricoloremission MIPs sensor, to evaluate its practical application to the determination of $\mathrm{BHb}$ in complicated biological fluids, as given in Table 1 . Since no $\mathrm{BHb}$ was detected in the urine samples, the 100-fold diluted urine samples were spiked with $\mathrm{BHb}$ at four concentration levels of $0.050,0.250,1.000$, and $2.000 \mu \mathrm{M}$. Recoveries of $99.25-111.7 \%$ with relative standard deviations (RSD) $<3.2 \%$ were obtained (Table 1 ), which revealed that the tricolor-emission sensor could be successfully applied in trace $\mathrm{BHb}$ detection in real samples. 
Table 1. Recoveries and Relative Standard Deviations (RSD, $n=3$ ) for BHb Detection in the Spiked 100-Fold Diluted Bovine Urine Samples by the Developed Tricolor-Emission MIPs Sensor

$\begin{array}{cclc}\text { bovine urine } & \text { spiked }(\mu \mathrm{M}) & \text { found }(\mu \mathrm{M}) & \text { recovery } \pm \text { RSD }(\%) \\ \text { sample 1 } & 0 & \text { not detected } & \text { not available } \\ & 0.050 & 0.052 & 104.0 \pm 1.8 \\ & 0.250 & 0.258 & 103.2 \pm 2.0 \\ 1.000 & 1.117 & 111.7 \pm 3.2 \\ \text { sample 2 } & 2.000 & 1.986 & 99.30 \pm 2.3 \\ & 0 & \text { not detected } & \text { not available } \\ & 0.050 & 0.053 & 106.0 \pm 2.1 \\ & 0.250 & 0.261 & 104.4 \pm 2.4 \\ & 1.000 & 1.089 & 108.9 \pm 3.0 \\ & 2.000 & 1.990 & 99.50 \pm 2.7\end{array}$

The construction strategy and sensing performance of the developed ternary-emission MIPs sensor for $\mathrm{BHb}$ detection were compared with that of the reported molecular imprinting fluorescence sensors. As given in Table 2, a series of fluorescent substances of $\mathrm{CdTe},{ }^{10,25,29,30}$ carbon dots (CDs), ${ }^{27}$ upconversion nanoparticles (UCNPs), ${ }^{31}$ and $\mathrm{Mn}$ doped $\mathrm{ZnS}^{32}$ were embedded into the imprinted polymers to provide $\mathrm{BHb}$-dosage-sensitive fluorescence intensity. In comparison with other fluorescent substances, CdTe QDs could provide more abundant color and narrower half-peak width, benefiting the construction of a multimodal fluorescence sensor without overlapping of peaks. In general, most of the multiemission MIPs fluorescence sensors ${ }^{10,25,32}$ achieve lower LODs and higher imprinting factors in comparison with the single-emission sensors. ${ }^{27,29,30}$ What is more, on the basis of the fluorescence intensity ratio change caused by the $\mathrm{BHb}$ concentration, it is easy to realize the visualization by observing the color variation of the multiemission sensors, ${ }^{10,25,32}$ whereas the single-emission sensors only provide an intensity reduction. ${ }^{27,29,30}$ However, only inconspicuous intensity changes are provided by the dual-emission UCNPs-containing MIPs, where both of the emission peaks are quenched by $\mathrm{BHb}{ }^{31}$ For our tricolor-emission sensor, the linear dynamic concentration range, LOD, and especially the imprinting factor are outstanding. Surprisingly, upon an increase in $\mathrm{BHb}$ and the resulting blue-emission enhancement but green- and redemission quenching, the fluorescence color varies from yellowish green to yellow to salmon to plum to purple and to finally blue. The color variation range is much larger than that in the aforementioned studies, ${ }^{10,25,27,29-32}$ as illustrated in Table 2: part A of the color evolution (yellowish green to plum) is equal to that of other green- and red-emission-based sensors (yellowish green to orange-red), and part B extends the color to the final blue, making the fluorescence images more colorful and $\mathrm{BHb}$ visualization more achievable. In addition, although it is similar to mixing g-CdTe@MIPs and r-CdTe@ $\mathrm{SiO}_{2}{ }^{23}$ the present post-imprinting mixing of the $\mathrm{b}$-, g-, and $\mathrm{r}$ MIPs in an appropriate ratio greatly simplifies the optimization of emission intensity ratio and therefore speeds up the experimental process and is more economical; more importantly, a higher imprinting factor and lower LOD are attained, suggesting higher selectivity and sensitivity.

Additionally, the sensitivity and selectivity of the MIPs sensor were also compared with those of antibody- or enzymebased biosensors. ${ }^{33-36}$ As seen in Table S1, our LOD is inferior by $0-2$ orders of magnitude because of the specificity $^{37}$ and supersensitivity of antibodies ${ }^{33-35}$ and enzymes; ${ }^{36}$ the high stability in a harsher environment and low cost of the MIPs, however, make possible their wide application in rapid on-site monitoring and frontline screening with an outstanding LOD down to a nanomolar level. The selectivity could be reflected by their practical applicability, because there are myriad species of interfering substances in real samples. As can be seen, the MIPs sensor also possesses a high anti-interference ability, like that of antibody or enzymebased biosensors. ${ }^{33-36}$ Although the functional monomer of APTES has universal application, without the specificity of antibodies and enzymes, the combined action of particular shape, size, and functional groups ensures the relatively high selectivity of the MIPs sensor.

\section{CONCLUSIONS}

To sum up, a novel tricolor-emission fluorescence MIPs sensor was successfully constructed by post-imprinting mixed b-, g-, and r-MIPs in an optimized ratio for the multiplexed and visual detection of $\mathrm{BHb}$. This ternary-emission sensor recognized $\mathrm{BHb}$ with turn-off green and red fluorescence along with the turn-on of blue fluorescence, therefore generating a remarkably profuse concentration-dependent color variation of the greenred-blue window. Integration of the superior selectivity of molecular imprinting, the ultrahigh sensitivity and inherent

Table 2. Comparison of the Construction Strategy and Sensing Performance with the Reported MIPs Fluorescence Sensors for BHb Detection

\begin{tabular}{|c|c|c|c|c|c|c|}
\hline sensor construction & emission mode & linear range & LOD & imprinting factor & fluorescence color variation & ref. \\
\hline CdTe@MIPs & single emission & $0.3-5.0 \mu \mathrm{M}$ & $69 \mathrm{nM}$ & 4.2 & & 29 \\
\hline M-CDs@MIPs & single emission & $0.05-16.0 \mu \mathrm{M}$ & $17.3 \mathrm{nM}$ & 4.1 & & 27 \\
\hline $\mathrm{SiO}_{2} @ \mathrm{CdTe} @ \mathrm{MIPs}$ & single emission & $0.02-2.1 \mu \mathrm{M}$ & $9.4 \mathrm{nM}$ & 3.79 & & 30 \\
\hline UCNPs@MOFs@MIPs & dual emission & $0.1-0.6 \mathrm{mg} / \mathrm{mL}$ & $0.062 \mathrm{mg} / \mathrm{mL}$ & 1.82 & & 31 \\
\hline Mn-doped ZnS@MIPs & dual emission & $0.1-10.0 \mu \mathrm{M}$ & $32 \mathrm{nM}$ & 3.1 & & 32 \\
\hline rCdTe@SiO & dual emission & $0.02-2.0 \mu \mathrm{M}$ & $6.3 \mathrm{nM}$ & 10.8 & & 10 \\
\hline gCdTe@MIPs +rCdTe@SiO & dual emission & $0.05-3 \mu \mathrm{M}$ & $9.6 \mathrm{nM}$ & 6.8 & & 25 \\
\hline b-MIPs + g-MIPs + r-MIPs & tricolor emission & $0.025-3 \mu \mathrm{M}$ & $7.8 \mathrm{nM}$ & 15.2 & Part B & this work \\
\hline
\end{tabular}

${ }^{a}$ Some references did not give the fluorescence color variation images, and the corresponding images presented in Table 2 were simulated by IE1931 chromaticity coordinate calculation according to the fluorescence spectra (for reference only). 
self-correction of multimodal fluorescence detection, and the operational convenience of the post-imprinting mixing strategy is notable. Specifically, this sensor did well in sensitivity and visualization, especially in comparison with the traditional single-/dual-emission MIPs sensors. Due to this successful proof of concept, we believe that the multimodal-emission fluorescence MIPs sensor can be further developed and applied to the rapid on-site visual detection of various target analytes in more fields.

\section{ASSOCIATED CONTENT}

\section{S Supporting Information}

The Supporting Information is available free of charge on the ACS Publications website at DOI: 10.1021/acs.analchem.9b00082.

Synthesis of $\mathrm{SiO}_{2}$ and QDs, calculation of $I_{\text {blue }} / I_{\text {green }} / I_{\text {red, }}$ characterization of SEM, TEM, EDX, and FT-IR, fluorescence spectra of one-pot-prepared MIPs, fluorescence intensities and images of dual emission/ triemission sensors, response time, absorbance spectrum of $\mathrm{BHb}$ and fluorescence spectrum of triemission MIPs, fluorescence spectra and images of single-emission MIPs, stability, and performance comparison with antibody or enzyme-based sensors (PDF)

\section{AUTHOR INFORMATION}

\section{Corresponding Authors}

*E-mail for H.X.: huaxiong100@126.com.

*E-mail for L.C.: 1xchen@yic.ac.cn.

\section{ORCID}

Lingxin Chen: 0000-0002-3764-3515

Notes

The authors declare no competing financial interest.

\section{ACKNOWLEDGMENTS}

This work was financially supported by the National Natural Science Foundation of China (21876199, 21575159, 21804010, 21667018, 31860470, 31660482, 41776110).

\section{REFERENCES}

(1) Fang, X. T.; Hultqvist, G.; Meier, S. R.; Antoni, G.; Sehlin, D.; Syvanen, S. NeuroImage 2019, 184, 881-888.

(2) Hoyos-Nogues, M.; Brosel-Oliu, S.; Abramova, N.; Munoz, F.; Bratov, A.; Mas-Moruno, C.; Gil, F. Biosens. Bioelectron. 2016, 86, 377-385.

(3) Ahn, Y. H.; Kim, Y.; Ji, E. S.; Lee, J. Y.; Jung, J.; Ko, J. H.; Yoo, J. S. Anal. Chem. 2010, 82, 4441-4447.

(4) Tawil, N.; Sacher, E.; Mandeville, R.; Meunier, M. Biosens. Bioelectron. 2012, 37, 24-29.

(5) Diaz-Fernandez, A.; Miranda-Castro, R.; De-los-Santos-Alvarez, N.; Fernandez Rodriguez, E.; Jesus Lobo-Castanon, M. Biosens. Bioelectron. 2019, 128, 83-90.

(6) Banerjee, S.; Koenig, B. J. Am. Chem. Soc. 2013, 135, 29672970.

(7) Chen, L.; Wang, X.; Lu, W.; Wu, X.; Li, J. Chem. Soc. Rev. 2016, $45,2137-2211$.

(8) Luan, J.; Xu, T.; Cashin, J.; Morrissey, J. J.; Kharasch, E. D.; Singamaneni, S. Anal. Chem. 2018, 90, 7880-7887.

(9) Bognar, J.; Szucs, J.; Dorko, Z.; Horvath, V.; Gyurcsanyi, R. E. Adv. Funct. Mater. 2013, 23, 4703-4709.

(10) Lu, H.; Xu, S. Talanta 2017, 165, 482-488.

(11) Yang, Q.; Li, J.; Wang, X.; Peng, H.; Xiong, H.; Chen, L. Biosens. Bioelectron. 2018, 112, 54-71.
(12) Li, Q.; Kamra, T.; Ye, L. Chem. Commun. 2016, 52, $12237-$ 12240.

(13) Chantada-Vázquez, M. P.; Sánchez-González, J.; Peña-Vázquez, E.; Tabernero, M. J.; Bermejo, A. M.; Bermejo-Barrera, P.; MoredaPiñeiro, A. Biosens. Bioelectron. 2016, 75, 213-221.

(14) Srikun, D.; Miller, E. W.; Dornaille, D. W.; Chang, C. J. J. Am. Chem. Soc. 2008, 130, 4596-4597.

(15) Yang, Q.; Li, J.; Wang, X.; Peng, H.; Xiong, H.; Chen, L. Sens. Actuators, B 2019, 284, 428-436.

(16) Li, M.; Liu, H.; Ren, X. Biosens. Bioelectron. 2017, 89, 899-905.

(17) Amjadi, M.; Jalili, R. Biosens. Bioelectron. 2017, 96, 121-126.

(18) Cai, Y.; You, J.; You, Z.; Dong, F.; Du, S.; Zhang, L. Biosens. Bioelectron. 2018, 99, 332-337.

(19) Silvi, S.; Credi, A. Chem. Soc. Rev. 2015, 44, 4275-4289.

(20) Wu, X.; Zhang, Z.; Li, J.; You, H.; Li, Y.; Chen, L. Sens. Actuators, B 2015, 211, 507-514.

(21) Gao, M. Y.; Kirstein, S.; Mohwald, H.; Rogach, A. L.; Kornowski, A.; Eychmuller, A.; Weller, H. J. Phys. Chem. B 1998, 102, 8360-8363.

(22) Liu, Y.; Yu, J. J. Colloid Interface Sci. 2010, 351, 1-9.

(23) Dabrowski, M.; Lach, P.; Cieplak, M.; Kutner, W. Biosens. Bioelectron. 2018, 102, 17-26.

(24) Xu, S.; Lu, H. Biosens. Bioelectron. 2015, 73, 160-166.

(25) Wang, X.; Yu, S.; Liu, W.; Fu, L.; Wang, Y.; Li, J.; Chen, L. ACS Sensors 2018, 3, 378-385.

(26) Zhang, W.; He, X.; Li, W.; Zhang, Y. Chem. Commun. 2012, 48, $1757-1759$

(27) Lv, P.; Xie, D.; Zhang, Z. Talanta 2018, 188, 145-151.

(28) You, F.; Zhou, Y.; Zhang, X.; Huang, Z.; Bi, L.; Zhang, Z.; Wen, J.; Chen, Y.; Jiang, G.; Zheng, M. Anal. Chem. 2006, 78, 7138-7144.

(29) Wang, X.; Yu, J.; Li, J.; Kang, Q.; Shen, D.; Chen, L. Sens. Actuators, B 2018, 255, 268-274.

(30) Li, D.; He, X.; Chen, Y.; Li, W.; Zhang, Y. ACS Appl. Mater. Interfaces 2013, 5, 12609-12616.

(31) Guo, T.; Deng, Q.; Fang, G.; Gu, D.; Yang, Y.; Wang, S. Biosens. Bioelectron. 2016, 79, 341-346.

(32) Tan, L.; Huang, C.; Peng, R.; Tang, Y.; Li, W. Biosens. Bioelectron. 2014, 61, 506-511.

(33) Ofori, J. A.; Hsieh, Y. P. Food Control 2018, 86, 19-26.

(34) Chen, H.; Wu, C.; Tsai, M.; Huang, Y.; Chen, S. Anal. Chem. 2012, 84, 8635-8641.

(35) Xue, Q.; Bian, C.; Tong, J.; Sun, J.; Zhang, H.; Xia, S. Biosens. Bioelectron. 2011, 26, 2689-2693.

(36) van Bommel, M. R.; de Jong, A.; Tjaden, U. R.; Irth, H.; van der Greef, J. J. Chromatogr. A 2000, 886, 19-29.

(37) Vessman, J.; Stefan, R. I.; Van Staden, J. F.; Danzer, K.; Lindner, W.; Burns, D. T.; Fajgelj, A.; Muller, H. Pure Appl. Chem. 2001, 73, 1381-1386. 